\title{
Optical Properties of Star-Shaped Gold Nanoparticles
}

\author{
Colleen L. Nehl, ${ }^{\dagger}$ Hongwei Liao, ${ }^{\ddagger}$ and Jason H. Hafner ${ }^{\star}, t, \neq$ \\ Department of Physics \& Astronomy, Department of Chemistry, Rice University, \\ Houston, Texas 77005
}

Received December 6, 2005; Revised Manuscript Received March 9, 2006

\begin{abstract}
Here we report the synthesis, structure, and optical properties of ca. $100 \mathrm{~nm}$ star-shaped gold nanoparticles. Single particle spectroscopy measurements revealed that these nanoparticles have multiple plasmon resonances resulting in polarization-dependent scattering with multiple spectral peaks, which correspond to the different tips on the star-shaped structure. The plasmon resonances were also found to be extremely sensitive to the local dielectric environment.
\end{abstract}

The size- and shape-dependent physical properties of inorganic nanoparticles provide tunable materials with broad potential applications, ${ }^{1}$ and the fabrication of structurally complex nanoparticles further enhances their functionality. For example, the synthesis of semiconductor nanowires with core/shell heterostructures creates electronic junctions within the nanowire that can act as tunable nanophotonic lightemitting diodes. ${ }^{2}$ The synthesis of branched quantum dots ${ }^{3}$ could enable studies of entangled quantum states and quantum information processing within individual nanoparticles. ${ }^{4}$ Metallic nanoparticles also benefit from the formation of complex structures. Magnetic "barcode" nanowires that contain periodic domains of $\mathrm{NiFe} / \mathrm{Cu}^{5}$ and $\mathrm{Pt} / \mathrm{Cu}^{6}$ offer tunable magnetic properties. Noble metal nanoparticles exhibit localized surface plasmon resonances resulting in strong optical extinction at visible wavelengths. The localized surface plasmon resonance (LSPR) enables applications including biological and chemical sensing, ${ }^{7-9}$ biological imaging labels, ${ }^{10-12}$ and nanoscale optical waveguides. ${ }^{13}$ The formation of more complex metal nanostructures, such as metallodielectric gold nanoshells ${ }^{14}$ and gold nanocages, ${ }^{15}$ shifts the LSPR resonance to the near-infrared (NIR) enabling significant diagnostic and therapeutic biomedical applications. ${ }^{16,17}$ Here we describe the synthesis and optical properties of branched star-shaped gold nanoparticles which incorporate polarization-dependent scattering with multiple spectral peaks and strong dielectric sensitivity into a single structure.

Due to the symmetric face-centered cubic lattice of gold nanoparticles, the formation of anisotropic structures requires a selective capping agent during growth. For example, room-

\footnotetext{
* To whom correspondence may be addressed. E-mail: hafner@ rice.edu

Department of Physics \& Astronomy.

$\doteqdot$ Department of Chemistry.
}

temperature synthesis of gold nanorods with $97 \%$ yield has recently been demonstrated by seed-mediated growth directed by the surfactant cetyltrimethylammonium bromide (CTAB), ${ }^{18,19}$ and the process can be scaled up to $100 \mathrm{~mL}$ batches. ${ }^{20}$ In addition to nanorods, a variety of anisotropic gold nanoparticle shapes, including cubes, prisms, and branched nanoparticles, can be fabricated using surfactants ${ }^{21-23}$ or other capping agents. ${ }^{24}$ In seed-mediated, surfactantdirected nanorod growth, surfactant-stabilized seed nanoparticles are synthesized through the reduction of gold chloride by sodium borohydride and then added to a gold chloride growth solution that also contains surfactant. We have found that when the surfactant-stabilized seed is replaced by a commercially available colloid (10 $\mathrm{nm}$ diameter gold colloid, Ted Pella), ca. $100 \mathrm{~nm}$ star-shaped gold nanoparticles are formed under the exact growth conditions that normally produce gold nanorods (see Supporting Information for details). Figure 1A displays a scanning electron microscopy (SEM) image of these gold "nanostars" deposited on a silicon substrate. These nanoparticles are distinct from those previously reported due to their relatively small size and complex three-dimensional structure. ${ }^{21-24}$ The bright points on the nanostars in the SEM image are tips pointing normal to the plane of the figure as confirmed by sample tilting (see Supporting Information). We have evaluated the yield of nanostar synthesis through SEM analysis of hundreds of particles. For each particle, the number of tips was counted, such that zero tips correspond to a spherical nanoparticle, and one, two, three, four, five, or six tips correspond to increasingly complex nanostars. During this analysis, we found a significant fraction of nanostars with small or poorly formed tips. We suspect these particles will not have the optical properties described below, so we counted them separately and refer to them simply as 

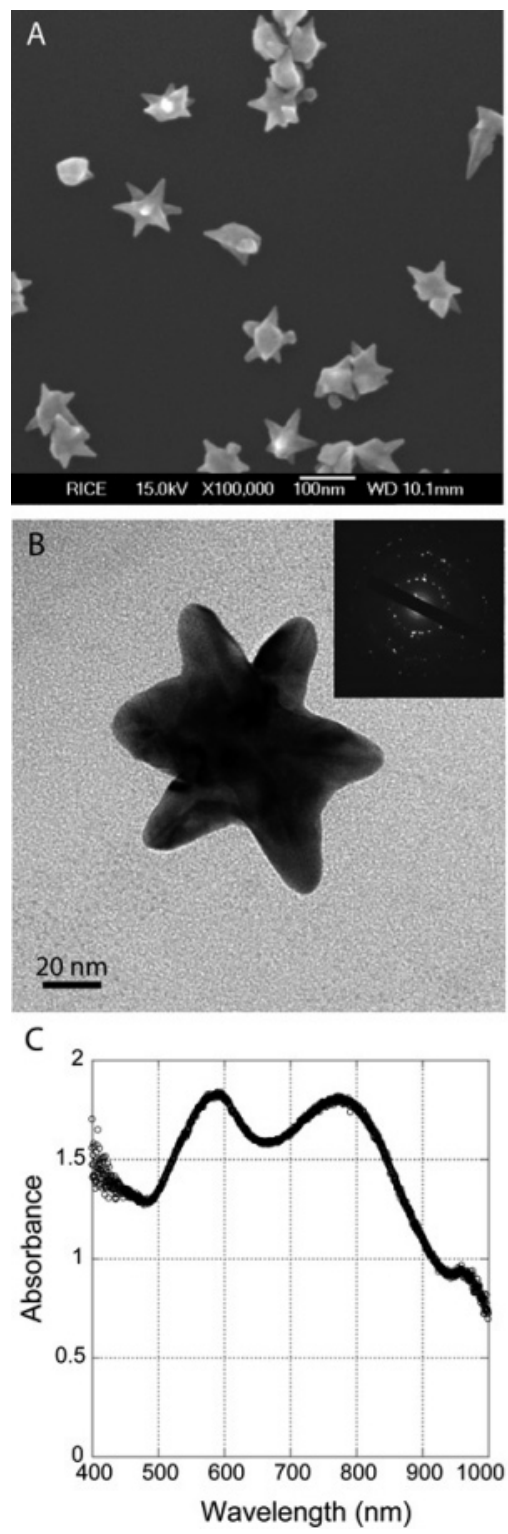

Figure 1. Star-shaped gold nanocrystals. SEM images reveal the structure and heterogeneity (A) while TEM and electron diffraction demonstrate that the nanostars have defects and consist of multiple crystal domains (B). An extinction spectrum of the nanostar solution exhibits broad visible and NIR peaks (C).

nanoparticles. Histograms of this yield analysis for a typical preparation with representative images are given in Figure 2B. Note that the analysis undercounts the number of tips on the nanostars since not all tips will be visible in the SEM images. For nanostar synthesis with commercial colloid seed, the yield of well-formed nanostars with at least three tips was about $14 \%$ of the particles.

We find the gold nanostar synthesis with commercial seed to be quite reproducible, having been successful with colloid from three separate shipments. However, the unknown surface chemistry of commercial nanoparticles makes it difficult to unravel the growth mechanism and guarantee future reproducibility. We have therefore developed a gold nanostar synthesis protocol from standard chemicals (see Supporting Information) that is also based on gold nanorod synthesis, ${ }^{18,19}$ but with an altered order of reactant mixing
A

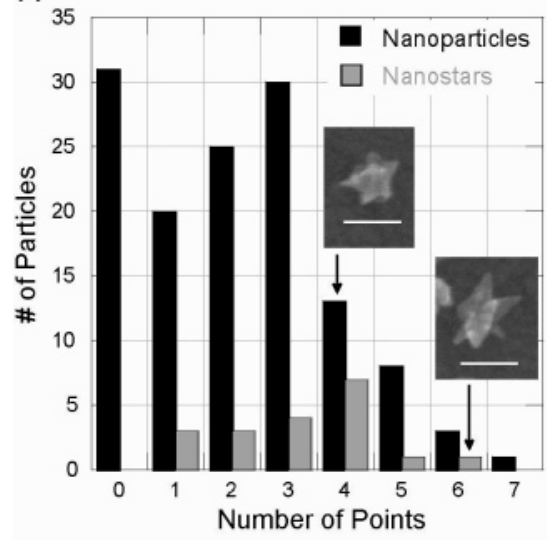

B

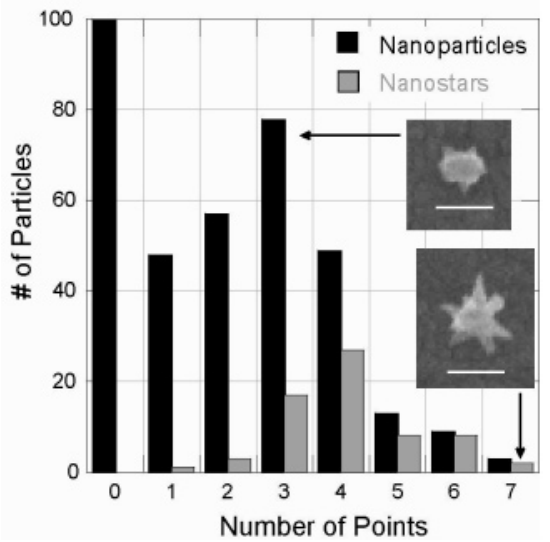

Figure 2. Star-shaped gold nanocrystal yield. SEM images were analyzed to determine yield in terms of the number of points per particle. Separate data are given for the surfactant-stabilized seed (A) and the commercial colloid seed (B). All scale bars are 100 nm.

and the addition of $\mathrm{NaOH}$. This method reproducibly synthesizes well-formed nanostars at $9 \%$ yield as defined above. The detailed yield data are shown in Figure 2A.

A transmission electron microscopy (TEM) image of a well-formed nanostar is presented in Figure 1B, where selected area electron diffraction (SAED) of that individual nanoparticle demonstrates that it consists of multiple crystal domains. Careful examination of Figure 1B reveals what appear to be twin defects along the nanostar tips (see the high-resolution figure in the Supporting Information). These defects are similar to those found in gold nanorods, where poor CTAB binding to twin defects is thought to be a source of growth anisotropy. ${ }^{25,26}$ Since nanostar and nanorod growth are both directed by CTAB surfactant, it follows that the nanostar tips may also grow due to poor CTAB binding. The nucleation of growth anisotropy at multiple sites on the nanostars could be due to a high defect density caused by rapid growth for both gold nanostar synthesis procedures. The commercial nanoparticle seeds may stimulate rapid growth due to their relatively unprotected surface chemistry compared to the surfactant-stabilized seed nanoparticles which produce gold nanorods. ${ }^{18,19}$ The addition of $\mathrm{NaOH}$ to the nanorod synthesis method may also lead to more rapid growth due to forced reduction of gold ions. ${ }^{21}$ The detailed 

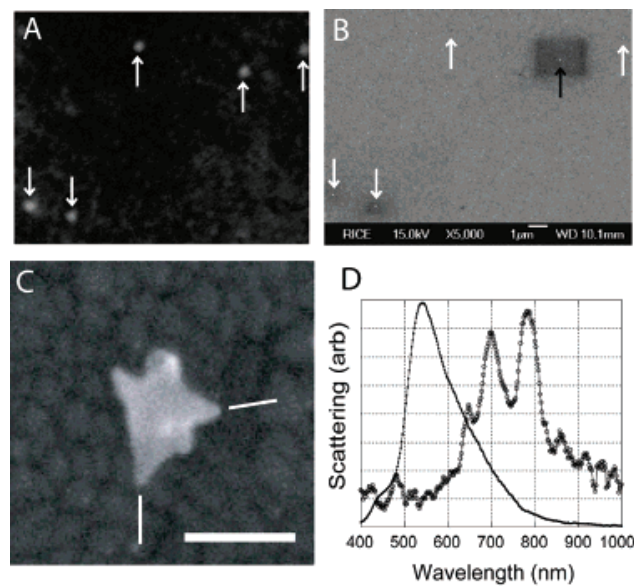

E
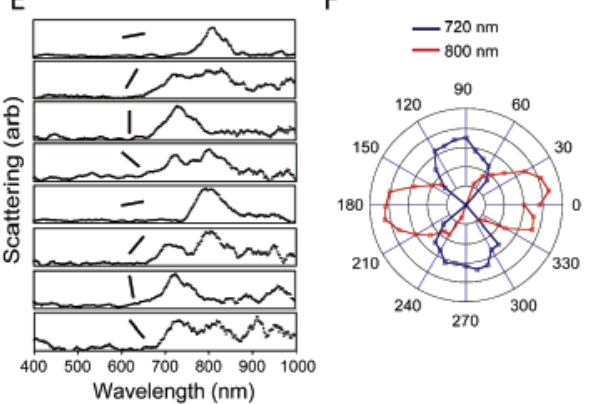

Figure 3. Optical (A) and SEM (B) images of a field of gold nanostars demonstrate the correlation of their position relative to alignment marks (not shown). The black arrow indicates the nanostar whose structure $(\mathrm{C}, 100 \mathrm{~nm}$ scale bar) and scattering spectrum (D, open circles) are displayed. The scattering spectrum of a $100 \mathrm{~nm}$ gold colloid is also plotted (D, points). Eight spectra at different analyzer angles are plotted (E) as well as the peak heights for the 720 and $800 \mathrm{~nm}$ peaks (F). Radial axis is arbitrary scattering.

mechanism of surfactant-directed growth of gold nanoparticles is under active investigation. ${ }^{27-30}$

Although the gold nanostar samples have marked structural heterogeneity, their extinction spectra exhibit broad yet welldefined peaks in the visible and NIR, as seen in Figure 1C. It has been well established theoretically and experimentally that elongated gold and silver nanoparticles feature a longitudinal plasmon resonance that red shifts from ca. 520 $\mathrm{nm}$ (the value for spherical gold nanoparticles) by an amount proportional to their aspect ratio. ${ }^{31,32}$ For example, gold nanorods with an aspect ratio of 3.3 (50 nm length by 15 $\mathrm{nm}$ diameter) have a plasmon resonance at $755 \mathrm{~nm}$ in water. ${ }^{20}$ The peak centered at $773 \mathrm{~nm}$ in Figure 1C is likely a similar longitudinal plasmon resonance due to the elongated tip structure of the nanostars. The $586 \mathrm{~nm}$ peak could represent the transverse plasmon resonance of the tips, which is also seen for gold nanorods, or the presence of large or slightly asymmetric spherical colloids in the solution.

Detailed characterization of the optical properties of complex, heterogeneous nanoparticles such as gold nanostars is best accomplished by single particle spectroscopy. ${ }^{33-41}$ The measurements presented here were carried out with dark field microscopy using both epi-illumination (0.9 NA, Figures 3 and 4 ) and transmitted light ( 0.75 NA oil immersion, Figures 5 and 6), a halogen light source, and an inverted optical
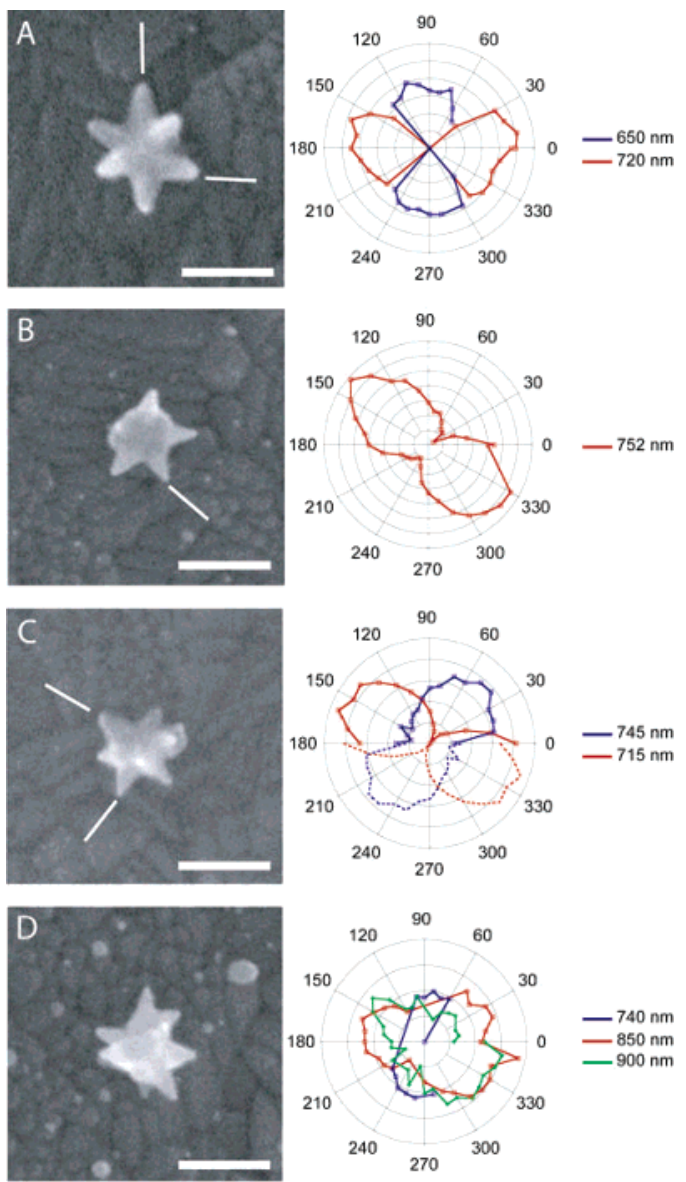

Figure 4. High-resolution structure and polarization-dependent scattering spectra for four gold nanostars. The radial axis is the scattered intensity with arbitrary units. The scale bars are $100 \mathrm{~nm}$. The data in (C) were only recorded over $180^{\circ}$ and are simply repeated to complete the plot.

microscope (Axiovert 200, Zeiss). The images of single nanoparticles were placed at the entrance of an imaging spectrometer (SpectraPro 150, Acton Research), and the spectra were detected with a thermoelectrically cooled CCD camera (PhotonMax 512, Roper Scientific). The spectra were normalized for spectral efficiency with a white reflectance standard (Edmund Industrial Optics). Note that single particle spectra recorded in this way represent only the scattering spectrum, rather than scattering plus absorption as for extinction measurements. Although the image in the optical microscope may have several diffraction limited spots, one cannot determine that each spot represents a single gold nanoparticle by optical imaging alone. ${ }^{33}$ We have therefore deposited the nanostars onto substrates with alignment marks created by evaporation through an enumerated TEM grid. This allows simultaneous optical and electron microscopic characterization to confirm that the spectra are recorded from a single nanoparticle and to reveal its detailed structure. Parts $\mathrm{A}$ and $\mathrm{B}$ of Figure 3 demonstrate this alignment (highresolution figures are available in the Supporting Information). Figure 3D presents the scattering spectrum for the gold nanostar shown in Figure 3C. The spectrum consists of three sharp peaks at 647, 700, and $783 \mathrm{~nm}$. Their positions and relative strengths suggest that the broad NIR peak in the 

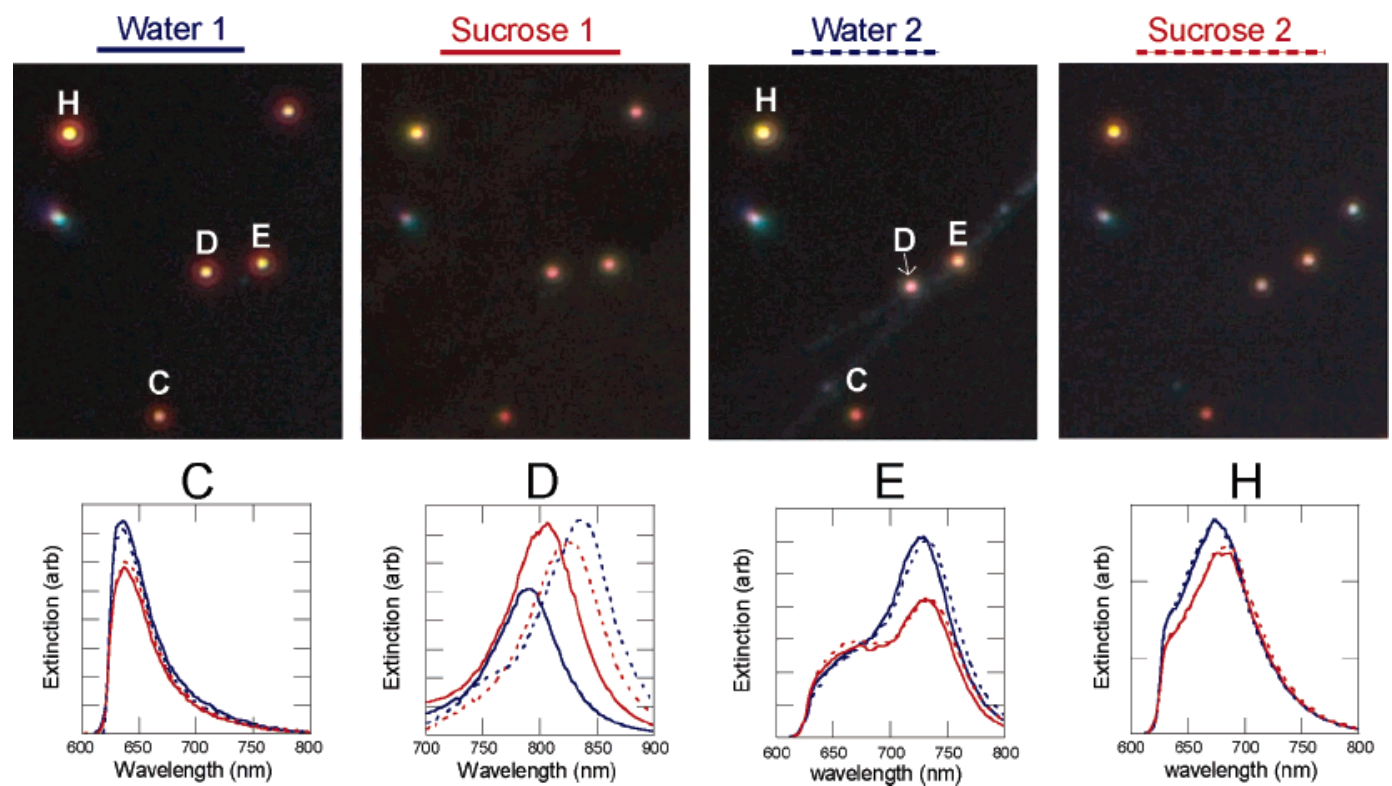

Figure 5. Gold nanostar dielectric shifts in water and sucrose. The top panels show dark field microscopy images of a field of nanostars which were taken from water, to sucrose, to water, and back again to sucrose. The bottom panel shows spectra of each particle in all four experiments. Note that the spectra of the three nanostars that do not translate are reproducible $(\mathrm{C}, \mathrm{E}$, and $\mathrm{H})$ while the spectra of one that does translate, and presumably rotates, is not (D).
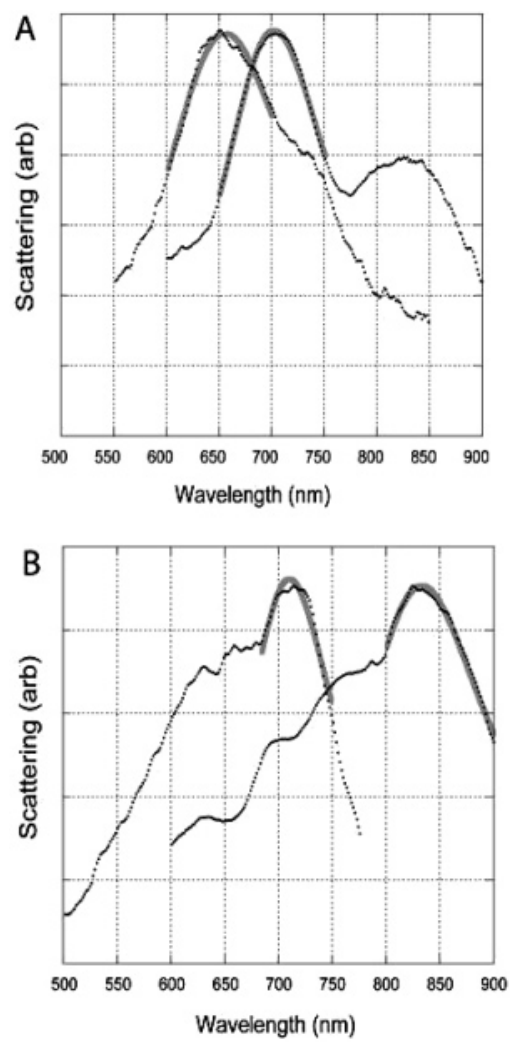

Figure 6. Single gold nanostar scattering spectra in water (left peak) and oil (right peak). The gray curve displays the Lorentzian curve fit. The nanostar in (A) has an LSPR shift of $649 \mathrm{meV} / \mathrm{RIU}$ and in (B) $1410 \mathrm{meV} / \mathrm{RIU}$.

extinction spectrum of the nanostar solution (Figure 1C) is a sum of several sharper peaks, as one would expect from a heterogeneous sample.

If the spectral peaks in Figure 3D are viewed as longitudinal plasmon resonances of the nanostar tips, with distinct resonant wavelengths due to the variable tip structure, one would expect each peak to be polarized at a different angle. To test this hypothesis, an analyzer was placed under the objective to determine the polarization dependence of the scattered light. As the analyzer rotates, different peaks grow and diminish, as illustrated in Figure 3E. Thirty six spectra at varying analyzer angles were recorded and fit to characterize the multiple peaks in each spectrum. The peak heights follow $\cos (\Theta)$ dependences with analyzer angle (Figure 3F), demonstrating that the scattered light is linearly polarized at a distinct angle for each peak and is in good agreement with the polarization dependence of the longitudinal resonance of gold nanorods. ${ }^{34}$ The polarization-dependent spectra for four more nanostars, along with their high-resolution structure, are presented in Figure 4, revealing similar multispectral polarized light scattering. Lines have been added to the SEM images that represent the polarization angle in order to assign spectral peaks to specific tips. Interestingly, the angle often matches the edge of a nanostar tip rather than a bisector to the tip. All tips for each nanostar are not represented in the spectrum due to several factors. First, if multiple peaks are at a similar wavelength, their angular separation is difficult to resolve due to significant spectral overlap. For example, three peaks were identified for the complicated nanostar in Figure 3D, yet none could be resolved into a simple $\cos (\Theta)$ dependence. Also, the small peak at $647 \mathrm{~nm}$ of Figure 2D could be seen at some analyzer angles but was usually lost in the larger $700 \mathrm{~nm}$ peak and could not be tracked. Second, some of the peaks could be beyond the range of the detector $(1000 \mathrm{~nm})$ or the wavelength at which the analyzer provides good polarization contrast $(900 \mathrm{~nm})$. Finally, these nanostars are three-dimensional objects. The orientation of the tips relative to the plane of observation will affect how efficiently they are excited and how efficiently their scattering is 
collected in a dark field imaging geometry. The smaller peaks in the spectrum in Figure 3D are likely due to such out-ofplane tips. The spectrum for a spherical $100 \mathrm{~nm}$ gold colloid recorded under the exact same conditions is also plotted for reference to demonstrate that the small peaks in the nanostar spectra are not noise or an artifact of the measurement.

We have investigated the sensitivity of the gold nanostar resonances to the dielectric environment by measuring their scattering spectra in water $(n=1.33)$, sucrose $(n=1.38)$, and immersion oil $(n=1.515)$. These LSPR shifts, usually reported in eV/RIU (shift in photon energy per change in refractive index unit), help quantify their applicability as biological and chemical LSPR sensors. ${ }^{89}$ LSPR shifts have been determined for gold nanospheres, ${ }^{42}$ silver triangles fabricated by nanosphere lithography, ${ }^{32}$ single silver nanoparticles, ${ }^{41,43,44}$ and gold nanoshells ${ }^{45,46}$ with values ranging from 500 to $800 \mathrm{meV} / \mathrm{RIU}$. LSPR shift measurements on gold nanostars can be complicated due to their threedimensional structure and multiple scattering peaks. If the nanostar were to rotate upon wetting or drying due to meniscus forces, an observed shift could be due to the alignment of a different tip with the microscope objective, rather than a true LSPR shift due to the altered dielectric environment. We first checked for this rotation effect by confirming the LSPR shift and monitoring the position of the nanoparticle upon several changes of solution. Figure 5 shows LSPR spectra and images for four nanostars in a field in water, then sucrose, then water, then sucrose. The substrate was dried between each measurement. The three nanostars that show no translation $(\mathrm{C}, \mathrm{E}$, and $\mathrm{H})$ have reproducible spectra. However, the nanostar that does translate, and therefore likely rotates, has nonreproducible spectra with no consistent shift.

Figure 6 shows LSPR shifts for two nanostars from water to oil. Neither of these nanostars translates, suggesting no rotation. Additional evidence for a lack of rotation is found in the consistent spectral shape of the LSPR in both media. The Lorentzian fits in Figure 6 indicate dielectric sensitivities of $649 \mathrm{meV} / \mathrm{RIU}$ and $1410 \mathrm{meV} / \mathrm{RIU}$ for the individual gold nanostars. It has been recently suggested that the spectral line width should also be considered when evaluating nanoparticles for LSPR sensing since it will affect the minimum shift that can be detected in a measurement. A figure of merit is therefore calculated which is simply the dielectric sensitivity described above divided by the line width of the plasmon resonance in $\mathrm{eV} .{ }^{43}$ The Lorentzian fits in Figure 6 have line widths of 0.17 and $0.13 \mathrm{eV}$, respectively. The figures of merit for the particles in Figure 6 are 3.8 and 10.7, respectively, the latter value being a factor of 2 higher than that previously observed for any other nanoparticle due to its high dielectric sensitivity.

The optical properties described above for gold nanostars provide enhanced functionality for several biological applications of noble metal nanostructures. Due to their bright, stable scattering signal, metallic nanoparticles are being pursued as labels for microscopic imaging. ${ }^{10}$ Anisotropic nanoparticles, such as gold nanorods, can reveal twodimensional nanoscale orientation through analysis of their polarized scattering, which could have significant applications for biological and materials research. ${ }^{11}$ The gold nanostars described here provide a multispectral signal from a single nanoparticle that could be used to detect threedimensional orientation by exploiting their multidirectional polarized scattering. In addition, the very strong dielectric sensitivity and sharp NIR resonances make these nanoparticles ideal substrates for LSPR sensors, a powerful yet simple biomedical sensing platform for the detection of low level analytes. ${ }^{9,47}$ These results demonstrate that progress in the synthesis of structurally complex noble metal nanoparticles will lead to enhanced properties and novel applications, as it has for semiconductor and magnetic nanomaterials.

Acknowledgment. The authors thank Wenhua Guo for assistance with the electron diffraction measurements. This work was supported by the U.S. Army Research Laboratory and the U.S. Army Research Office under Grant Number W911NF-04-1-0203, the Nanoscale Science and Engineering Initiative of the National Science Foundation under NSF Award No. EEC-018007, and the Welch Foundation under Grant Number C-1556.

Supporting Information Available: The synthesis protocols for preparing star-shaped gold nanoparticles, tilted SEM image to demonstrate three-dimensional structure, highresolution TEM image from Figure 1, high-resolution optical and SEM alignment images from Figure 3 This material is available free of charge via the Internet at http://pubs.acs.org.

\section{References}

(1) Rotello, V. M. Nanoparticles: building blocks for nanotechnology. Kluwer Academic/Plenum Publishers: New York, 2004.

(2) Qian, F.; Gradecak, S.; Li, Y.; Wen, C.-Y.; Lieber, C. M. Nano Lett. 2005, 5 (11), 2287-2291.

(3) Milliron, D. J.; Hughes, S. M.; Cui, Y.; Manna, L.; Li, J. B.; Wang, L. W.; Alivisatos, A. P. Nature 2004, 430 (6996), 190-195.

(4) Bayer, M.; Hawrylak, P.; Hinzer, K.; Fafard, S.; Korkusinski, M.; Wasilewski, Z. R.; Stern, O.; Forchel, A. Science 2001, 291 (5503), 451-453.

(5) Dubois, S.; Marchal, C.; Beuken, J. M.; Piraux, L.; Duvail, J. L.; Fert, A.; George, J. M.; Maurice, J. L. Appl. Phys. Lett. 1997, 70 (3), 396-398.

(6) Choi, J.-R.; Oh, S. J.; Ju, H.; Cheon, J. Nano Lett. 2005, 5 (11), 2179-2183.

(7) Elghanian, R.; Storhoff, J. J.; Mucic, R. C.; Letsinger, R. L.; Mirkin, C. A. Science 1997, 277 (5329), 1078-1081.

(8) Haynes, C. L.; McFarland, A. D.; Van Duyne, R. P. Anal. Chem. 2005, 77 (17), 338A-346A.

(9) Haes, A. J.; Hall, W. P.; Chang, L.; Klein, W. L.; Van Duyne, R. P. Nano Lett. 2004, 4 (6), 1029-1034.

(10) Schultz, S.; Smith, D. R.; Mock, J. J.; Schultz, D. A. Proc. Natl. Acad. Sci. U.S.A. 2000, 97 (3), 996-1001.

(11) Sönnichsen, C.; Alivisatos, A. P. Nano Lett. 2005, 5 (2), 301-304.

(12) Wang, H.; Huff, T. B.; Zweifel, D. A.; He, W.; Low, P. S.; Wei, A.; Cheng, J.-X. Proc. Natl. Acad. Sci. U.S.A. 2005, 102 (44), 1575215756.

(13) Maier, S. A.; Kik, P. G.; Atwater, H. A.; Meltzer, S.; Harel, E.; Koel, B. E.; Requicha, A. A. G. Nat. Mater. 2003, 2 (4), 229-232.

(14) Oldenburg, S. J.; Averitt, R. D.; Westcott, S. L.; Halas, N. J. Chem. Phys. Lett. 1998, 288, 243-247.

(15) Chen, J.; Saeki, F.; Wiley, B.; Cang, H.; Cobb, M.; Li, Z.; Au, L.; Zhang, H.; Kimmey, M.; Li, X.; Xia, Y. Nano Lett. 2005, 5 (3), $473-477$.

(16) Loo, C.; Lowery, A.; Halas, N.; West, J.; Drezek, R. Nano Lett. 2005, 5 (4), 709-711.

(17) Huang, X.; El-Sayed, I. H.; Qian, W.; El-Sayed, M. A. J. Am. Chem. Soc. 2006, 128, 2115-2120. 
(18) Nikoobakht, B.; El-Sayed, M. A. Chem. Mater. 2003, 15 (10), 19571962.

(19) Sau, T. K.; Murphy, C. J. Langmuir 2004, 20 (15), 6414-6420.

(20) Liao, H.; Hafner, J. Chem. Mater. 2005, 17 (18), 4636-4641.

(21) Chen, S.; Wang, Z.; Ballato, J.; Foulger, S.; Carroll, D. J. Am. Chem. Soc. 2003, 125 (52), 16186-16187.

(22) Hao, E.; Bailey, R.; Schatz, G.; Hupp, J.; Li, S. Nano Lett. 2004, 4 (2), 327-330.

(23) Sau, T. K.; Murphy, C. J. J. Am. Chem. Soc. 2004, 126 (28), 86488649.

(24) Yamamoto, M.; Kahiwagi, Y.; Sakata, T.; Mori, H.; Nakamoto, M. Chem. Mater. 2005, 17 (22), 5391-5393.

(25) Johnson, C. J.; Dujardin, E.; Davis, S. A.; Murphy, C. J.; Mann, S. J.f Mater. Chem. 2002, 12 (6), 1765-1770.

(26) Gai, P. L.; Harmer, M. A. Nano Lett. 2002, 2 (7), 771-774.

(27) Orendorff, C. J.; Murphy, C. J. J. Phys. Chem. B 2006, ASAP

(28) Chen, H. M.; Peng, H. C.; Liu, R. S.; Asakura, K.; Lee, C. L.; Lee, J. F.; Hu, S. F. J. Phys. Chem. B 2005, 109 (42), 19553-19555.

(29) Gou, L. F.; Murphy, C. J. Chem. Mater. 2005, 17 (14), 3668-3672.

(30) Liu, M.; Guyot-Sionnest, P. J. Phys. Chem. B 2005, 109 (47), $22192-$ 22200.

(31) Link, S.; Mohamed, M.; El-Sayed, M. J. Phys. Chem. B 1999, 103 (16), 3073-3077.

(32) Haes, A. J.; Zou, S. L.; Schatz, G. C.; Van Duyne, R. P. J. Phys. Chem. B 2004, 108 (22), 6961-6968.

(33) Nehl, C.; Grady, N.; Goodrich, G.; Tam, F.; Halas, N.; Hafner, J. Nano Lett. 2004, 4 (12), 2355-2359.

(34) Sönnichsen, C.; Franzl, T.; Wilk, T.; von Plessen, G.; Feldmann, J. Phys. Rev. Lett. 2002, 88 (7), 077402.
(35) Klar, T.; Perner, M.; Grosse, S.; von Plessen, G.; Spirkl, W.; Feldmann, J. Phys. Rev. Lett. 1998, 80 (19), 4249-4252.

(36) Krenn, J. R.; Schider, G.; Rechberger, W.; Lamprecht, B.; Leitner, A.; Aussenegg, F. R.; Weeber, J. C. Appl. Phys. Lett. 2000, 77 (21), 3379-3381.

(37) Liau, Y. H.; Unterreiner, A. N.; Chang, Q.; Scherer, N. F. J. Phys. Chem. B 2001, 105 (11), 2135-2142.

(38) Lindfors, K.; Kalkbrenner, T.; Stoller, P.; Sandoghdar, V. Phys. Rev. Lett. 2004, 93 (3).

(39) Michaels, A. M.; Jiang, J.; Brus, L. J. Phys. Chem. B 2000, 104 (50), 11965-11971.

(40) Mock, J. J.; Barbic, M.; Smith, D. R.; Schultz, D. A.; Schultz, S. J.f Chem. Phys. 2002, 116 (15), 6755-6759.

(41) Mock, J. J.; Smith, D. R.; Schultz, S. Nano Lett. 2003, 3 (4), 485491.

(42) Underwood, S.; Mulvaney, P. Langmuir 1994, 10 (10), 3427-3430.

(43) Sherry, L. J.; Chang, S.-H.; Schatz, G. C.; van Duyne, R. P. Nano Lett. 2005, 5 (10), 2034-2038.

(44) McFarland, A. D.; Van Duyne, R. P. Nano Lett. 2003, 3, 10571062.

(45) Sun, Y.; Xia, Y. Anal. Chem. 2002, 74, 5297-5305.

(46) Tam, F.; Moran, C.; Halas, N. J. Phys. Chem. B 2004, 108 (45), 17290-17294.

(47) Haes, A. J.; Van Duyne, R. P. J. Am. Chem. Soc. 2002, 124 (35), 10596-10604.

NL052409Y 